\title{
Alterations in sarcomere function modify the hyperplastic to hypertrophic transition phase of mammalian cardiomyocyte development
}

\author{
Benjamin R. Nixon, ${ }^{1}$ Alexandra F. Williams, ${ }^{1}$ Michael S. Glennon, ${ }^{1}$ Alejandro E. de Feria, ${ }^{1}$ \\ Sara C. Sebag, ${ }^{1}$ H. Scott Baldwin, ${ }^{2,3}$ and Jason R. Becker ${ }^{1,4}$ \\ 'Department of Medicine, Division of Cardiovascular Medicine, 2Department of Pediatrics, Division of Pediatric Cardiology, \\ ${ }^{3}$ Department of Cellular and Developmental Biology, ${ }^{4}$ Department of Molecular Physiology and Biophysics, Vanderbilt \\ University School of Medicine, Nashville, Tennessee, USA.
}

\begin{abstract}
It remains unclear how perturbations in cardiomyocyte sarcomere function alter postnatal heart development. We utilized murine models that allowed manipulation of cardiac myosin-binding protein C (MYBPC3) expression at critical stages of cardiac ontogeny to study the response of the postnatal heart to disrupted sarcomere function. We discovered that the hyperplastic to hypertrophic transition phase of mammalian heart development was altered in mice lacking MYBPC3 and this was the critical period for subsequent development of cardiomyopathy. Specifically, MYBPC3-null hearts developed evidence of increased cardiomyocyte endoreplication, which was accompanied by enhanced expression of cell cycle stimulatory cyclins and increased phosphorylation of retinoblastoma protein. Interestingly, this response was self-limited at later developmental time points by an upregulation of the cyclin-dependent kinase inhibitor p21. These results provide valuable insights into how alterations in sarcomere protein function modify postnatal heart development and highlight the potential for targeting cell cycle regulatory pathways to counteract cardiomyopathic stimuli.
\end{abstract}

Authorship note: B.R. Nixon and A.F. Williams are co-first authors.

Conflict of interest: The authors have declared that no conflict of interest exists.

Submitted: September 13, 2016 Accepted: January 13, 2017 Published: February 23, 2017

\section{Reference information:} JCI Insight. 2017;2(4):e90656. https:// doi.org/10.1172/jci.insight.90656.

\section{Introduction}

Sarcomere gene mutations are a common genetic cause of cardiomyopathy in human populations (1). Mutation carriers can exhibit a wide spectrum of disease phenotypes that include hypertrophic cardiomyopathy, dilated cardiomyopathy, and restrictive cardiomyopathy (2). Interestingly, human sarcomeric cardiomyopathies can manifest at many different developmental time periods. Mutation burden is proposed to be one reason for this variation in disease presentation, and humans with homozygous or compound heterozygous sarcomere gene mutations are reported to have a more fulminant form of cardiomyopathy compared with heterozygous carriers (3-5). Likewise, animal models that are homozygous or compound heterozygous for sarcomere gene mutations also develop evidence of cardiomyopathy at earlier developmental time points and the cardiomyopathy is more severe compared with heterozygous animals $(6,7)$. A consistent finding across multiple animal models of sarcomeric cardiomyopathy is that the early postnatal time period is important for both cardiomyopathy development and prevention of disease. Prevention of structural and functional defects associated with cardiomyopathy development has been demonstrated in murine models by viral reintroduction of wild-type protein and modulation of mutant RNA splicing or expression (8-11). However, it remains unclear why this developmental time period is important in the pathogenesis of sarcomeric cardiomyopathies.

To uncover the mechanisms underlying cardiomyopathy development in the early postnatal heart, we utilized murine models with germline deletion, conditional deletion, and conditional reactivation of the sarcomere protein cardiac myosin-binding protein C (MYBPC3). We discovered that disruptions in sarcomere function altered the hyperplastic to hypertrophic transition phase of postnatal murine heart development by modifying multiple cardiomyocyte cell cycle regulatory pathways. Importantly, selective targeting of these pathways was performed to confirm the critical role that dysregulated cardiomyocyte 
cell cycle activity has in the pathogenesis of this disease. This study provides unique insights into the role of cardiomyocyte endoreplication and cell cycle regulatory pathways in modifying the cardiomyocyte response to pathogenic stimuli and provides potentially therapeutic therapeutic targets for prevention of sarcomeric cardiomyopathies.

\section{Results}

Lack of MYBPC3 results in rapid development of postnatal cardiomyopathy. To delineate the role of altered sarcomeric function on heart development, we created murine models with germline deletion, conditional deletion, or conditional reactivation of MYBPC3 (Supplemental Figure 1; supplemental material available online with this article; https://doi.org/10.1172/jci.insight.90656DS1). We first examined germline MYBPC3-null animals and discovered that there were no detectable differences in heart structure at P2; however, by $\mathrm{P} 7$ there were clear changes in left ventricular (LV) size and heart mass (Figure 1, A-C). These cardiac structural changes were even more pronounced at later stages of postnatal heart development, P15 and P25 (Figure 1, A-C). In contrast to the germline MYBPC3-null animals, MYBPC3 heterozygous animals did not develop changes in cardiac structure compared with age-matched control animals. Similar to heart mass, P2 cardiac systolic function was similar between control and null mice, while systolic function decreased substantially by P25 in null mice (Figure 1, D-F). Furthermore, an increase in both interventricular septal thickness at end-diastole (IVSd) and LV posterior wall thickness at end-diastole (LVPWd) at P25 was observed (Figure 1, G and H).

Deletion of MYBPC3 in the adult does not induce rapid development of cardiomyopathy. To better evaluate the role of postnatal cardiac development in MYBPC3 cardiomyopathy, we used a murine model that allows conditional deletion of MYBPC3 protein (Figure 1I and Supplemental Figure 1A). We injected these mice with tamoxifen at P30 and then analyzed the mice at P90. Despite the complete elimination of MYBPC3 protein, the conditional MYBPC3-null (Null C) animals had no detectable changes in heart mass (Figure $1, \mathrm{I}-\mathrm{K})$. Additionally, we observed no decline in fractional shortening in the Null C mice relative to control mice (Figure 1, L and M). We also measured myocardial Nppb gene expression in the Null $\mathrm{C}$ animals (Supplemental Figure 2A). In contrast to the large increase in Nppb expression exhibited in the P25 germline MYBPC3-null animals (P25 Null), there were minimal changes in Nppb gene expression in the adult Null C animals (P90 Null C). These data show that the early postnatal heart and adult heart have divergent responses to elimination of MYBPC3 protein expression.

Cardiomyopathy can be prevented with the early reintroduction of $M Y B P C 3$. We developed a mouse line that allows for the conditional reactivation of MYBPC3 protein production (Figure 2A and Supplemental Figure 1B). Without administration of tamoxifen, the MYBPC3 reactivation line (Null R) was identical to the germline MYBPC3-null animals and rapidly developed a postnatal cardiomyopathy (Supplemental Figure 2, B-D). However, when tamoxifen was administered at P2 (Null R-P2), cardiomyopathy was effectively prevented (Figure 2, B-F). In addition to changes in cardiac structure and systolic function, we also confirmed that $\mathrm{P} 2$ reactivation of MYBPC3 protein production also prevented changes in cardiomyocyte hypertrophy as measured by cardiomyocyte cross-sectional area (Figure 2, B and G).

To determine if our tamoxifen dose created a uniform conversion from the MYBPC3-null allele to the MYBPC3-reactivation allele we injected different concentrations of tamoxifen in the Null R-P2 animals. Since the MYBPC3 Tm1a-null allele is tagged with a lacZ insert (Supplemental Figure 1B), this allowed us to determine what percentage of cardiomyocytes converted from $\beta$-gal positive (Mybpc $3^{\text {tmla } / \mathrm{tmla}}$ or Myb$\mathrm{pc} 3^{\mathrm{tmla} / \mathrm{tm} 1 \mathrm{c}}$ ) to $\beta$-gal negative $\left(\mathrm{Mybpc} 3^{\mathrm{tm} 1 \mathrm{c} / \mathrm{tm} 1 \mathrm{c}}\right.$ ) after administration of tamoxifen. The $200 \mathrm{mg} / \mathrm{kg}$ dose of tamoxifen caused complete conversion from $\beta$-gal-positive to $\beta$-gal-negative cardiomyocytes (Supplemental Figure 2, E and F). In contrast, the lower doses of tamoxifen $(25 \mathrm{mg} / \mathrm{kg}$ and $12.5 \mathrm{mg} / \mathrm{kg})$ caused considerably fewer cardiomyocytes to become $\beta$-gal negative (Supplemental Figure 2, E and F). Likewise, there was a corresponding reduction in MYBPC3 protein expression in the myocardium of Null $\mathrm{R}$ mice that received lower doses of tamoxifen (Figure $2 \mathrm{H}$ ). Interestingly, despite lower doses of tamoxifen creating a mosaic population of MYBPC3-expressing cardiomyocytes, it was still possible to at least partially prevent the increase in heart mass in MYBPC3-null animals (Figure 2, I and J).

To determine if we could reverse the development of already established cardiomyopathy, we reactivated MYBPC3 protein production at later developmental time points (Figure 3A). Reactivation of MYBPC3 protein production at P7 (Null R-P7) was successful in normalizing heart mass by P25 (Figure 3, B-D). In contrast, reactivation of $\mathrm{MYBPC} 3$ protein production at $\mathrm{P} 30$ was unable to cause significant reductions 
A

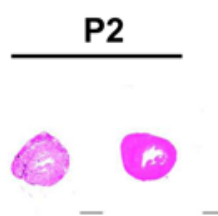

Ctl Null
P7

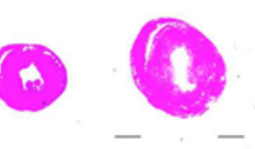

Ctl

Null

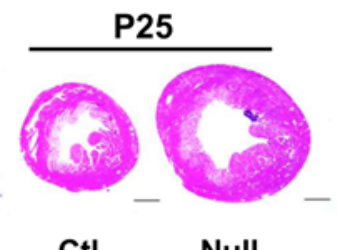

Ctl

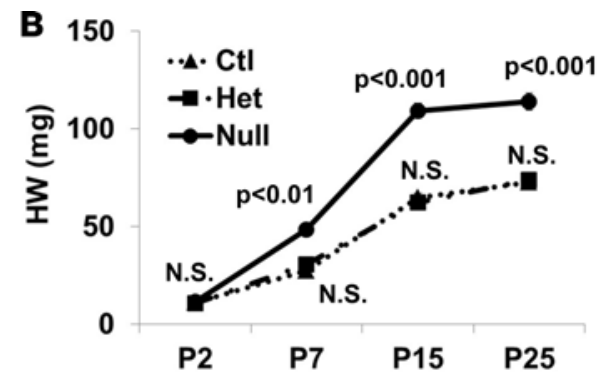

P2

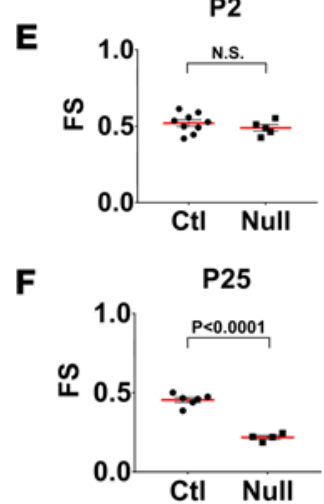

G

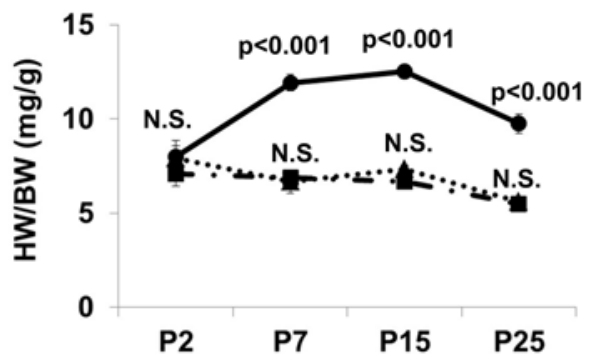

H
D

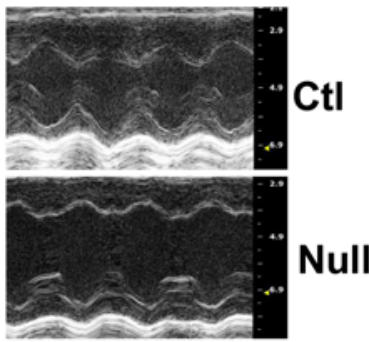

I
Conditional Deletion

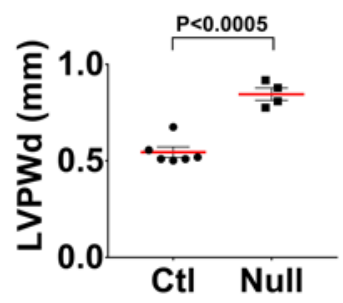

Tg(Myh6-cre/Esr1*)1Jmk

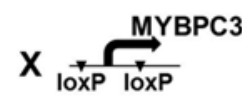

P30 \Tamoxifen inj.

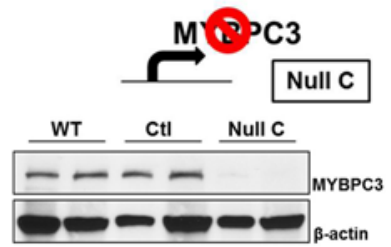

M

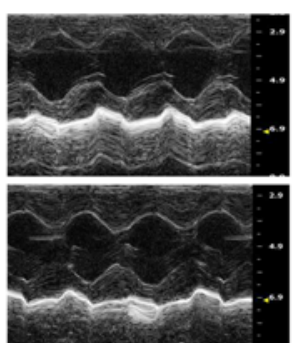

Ctl

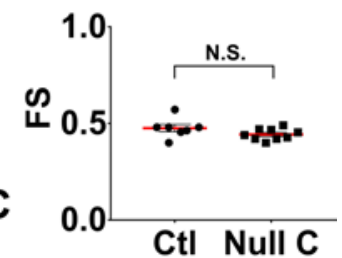

Figure 1. Lack of MYBPC3 results in rapid development of postnatal cardiomyopathy. (A) H\&E-stained cross sections of control (CtI) and MYBPC3null (Null) hearts at P2, P7, and P25. Scale bars: 1 mm. (B and C) Heart weight (HW) (B) and HW to body weight ratio (HW/BW) (C) of wild-type (Ctl, $n$ = 7), MYBPC3 heterozygous (Het, $n=9$ ), and MYBPC3-null (Null, $n=7$ ) hearts. (D) Example M-mode echocardiography of Ctl and Null mice at P25. (E and F) Fractional shortening (FS) of P2 CtI $(n=9)$ and Null $(n=5)(\mathbf{E})$ and P25 CtI $(n=6)$ and Null $(n=4)(\mathbf{F})$ mice. (G) Interventricular septal thickness at end diastole (IVSd) of P25 CtI $(n=6)$ and Null $(n=4)$ mice. (H) Left ventricular posterior wall thickness at end diastole (LVPWd) of P25 CtI ( $n$ $=6)$ and Null $(n=4)$ mice. (I) Schematic illustration of the transgenic line that allows MYBPC3 conditional deletion (Null C) after administration of tamoxifen at P30 (top). Western blot confirms that MYBPC3 protein expression was successfully eliminated in Null C mice at P90 (bottom). (J and K) HW (J) and HW to tibia length ratio (HW/TL) (K) in CtI $(n=11)$ and Null C $(n=12)$ mice. (L) Example M-mode echocardiography images of CtI and Null C mice at Pgo. (M) FS of CtI $(n=7)$ and Null C $(n=9)$ mice at P90. All results are shown as mean \pm SEM. Statistical analysis performed using an unpaired, 2-tailed Student's $t$ test. N.S., not significant. 
A

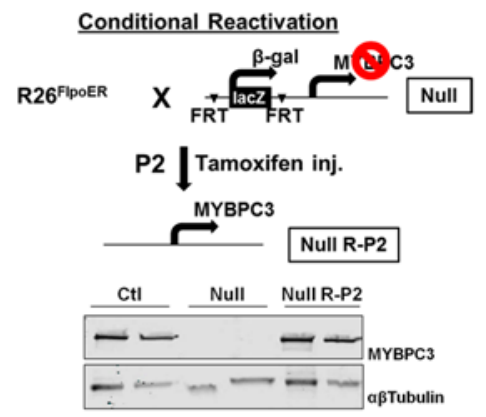

B

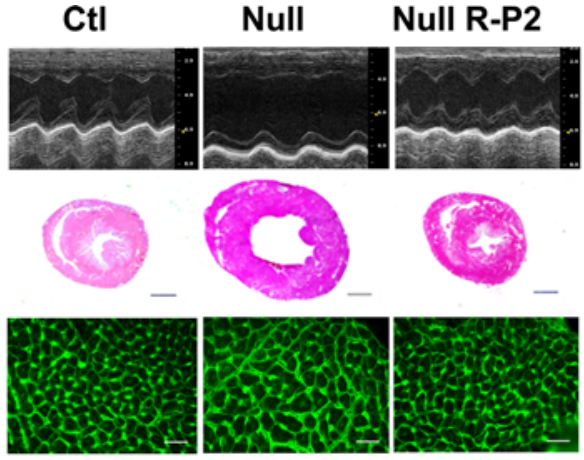

C

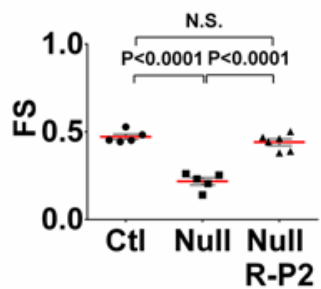

$\mathbf{F}$

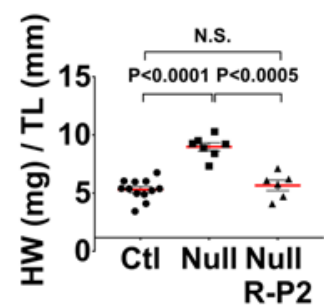

I

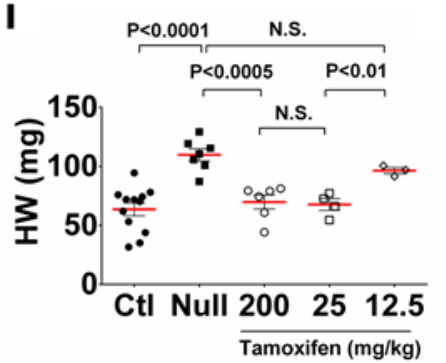

D

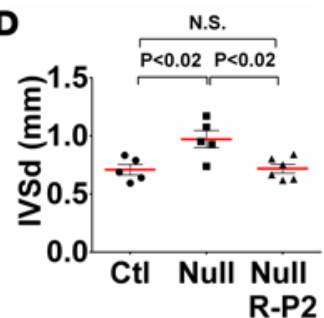

G

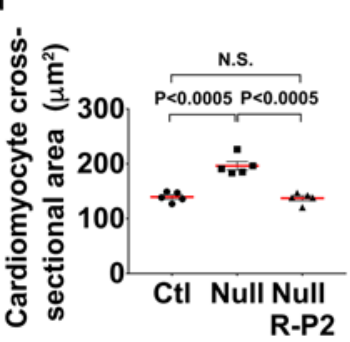

J

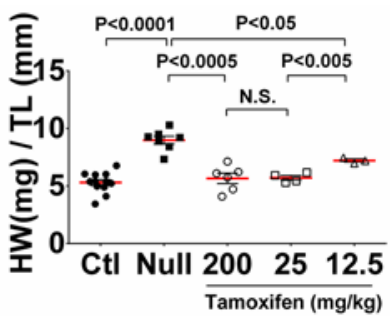

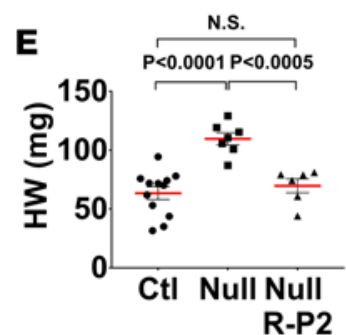

H

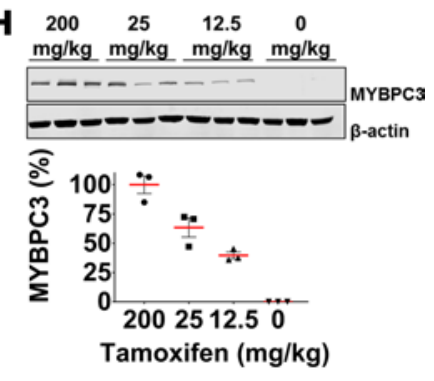

Figure 2. Cardiomyopathy can be prevented with early reintroduction of MYBPC3. (A) Schematic illustration of the transgenic line that allows conditional reactivation of MYBPC3 after administration of tamoxifen (Null R) (top). Western blot demonstrating successful reactivation of MYBPC3 protein expression at P10 in Null R mice injected with tamoxifen at P2 (Null R-P2) (bottom). (B) Phenotypic assessment of control (CtI), MYBPC3-null (Null), and Null R-P2 hearts at P25 as determined by M-mode echocardiography (top), $\mathrm{H} \& \mathrm{E}$-stained heart cross sections (middle, scale bar: $1 \mathrm{~mm}$ ), and wheat-germ agglutinin (WCA) immunohistochemical staining (bottom, scale bar: $30 \mu \mathrm{m}$ ). (C and D) Fractional shortening (FS) (C) and interventricular septal thickness diastole at end diastole (IVSd) (D) of 25-day-old CtI $(n=5)$, Null $(n=5)$, and Null R-P2 $(n=6)$ mice. (E and F) Heart weight (HW) (E) and HW to tibia length ratio (HW/TL) (F) of 25-day-old Ctl $(n=12)$, Null $(n=7)$, and Null R-P2 $(n=6)$ hearts. (G) Cardiomyocyte cross-sectional area of 25-day-old CtI $(n=5)$, Null $(n=5)$, and Null R-P2 $(n=5)$ hearts as assessed by WGA staining of left ventricle cross sections. (H) Western blot of MYBPC3 protein levels from conditional reactivation mice administered different doses of tamoxifen (top). Percentage of МYBPC3 protein relative to $200 \mathrm{mg} / \mathrm{kg}$ tamoxifen dose (normalized to $\beta$-actin) (bottom) ( $n=3 /$ group). (I and J) P25 HW (I) and HW/TL (J) of CtI $(n=12)$, Null ( $n=7)$, and conditional reactivation mice administered different doses of tamoxifen: $200 \mathrm{mg} / \mathrm{kg}(n=6), 25 \mathrm{mg} / \mathrm{kg}$ tamoxifen $(n=4), 12.5 \mathrm{mg} / \mathrm{kg}$ tamoxifen $(n=3)$ at P2. All results are shown as mean \pm SEM. Statistical analysis performed using an unpaired, 2-tailed Student's $t$ test. N.S., not significant.

in cardiac mass by P60 (Figure 3, E-H and Supplemental Figure $2 \mathrm{G})$. These results reinforce the importance of the early postnatal period in modifying the pathogenesis of MYBPC 3 cardiomyopathy.

MYBPC3 cardiomyopathy alters the hyperplastic to hypertrophic transition phase of heart development. The MYBPC3-null animals developed evidence of cardiomyopathy over a rapid period from P2 to P7. During this same developmental period, murine cardiomyocytes transition from hyperplastic to hypertrophic growth (12). Therefore, we assessed whether this hyperplastic to hypertrophic growth phase of murine heart development was altered in the MYBPC3-null animals. First, we examined the degree of cardiomyocyte DNA synthesis using Ki67 staining and discovered that MYBPC3-null cardiomyocytes had evidence of increased cardiomyocyte DNA synthesis at P7 and P25 compared with control animals (Figure 4, A and B, and Supplemental Figure 3B). Phospho-histone $\mathrm{H} 3$, a marker of mitosis, was also increased in MYBPC3-null cardiomyocytes from $\mathrm{P} 7$ and $\mathrm{P} 25$ animals (Figure 4C and Supplemental Figure 3, A and C). Synthesis of DNA and mitosis can occur independently of cardiomyocyte cytokinesis (karyokinesis without cytokinesis) Therefore, we then assessed whether there was evidence of aurora $B$ kinase staining in cardiomyocyte cleavage furrows, which is suggestive of active cardiomyocyte cytokinesis (Figure 4D). Importantly, we were able to accurately exclude non-cleavage furrow cardiomyocyte aurora B staining (Figure 4D) and aurora $B$ cleavage furrow staining in noncardiomyocyte populations (Figure 4D). Interestingly, we found that there was no difference in aurora B kinase cleavage furrow staining in MYBPC3-null or control cardiomyocytes at P2, P7, and P25 (Figure 4E). 
A

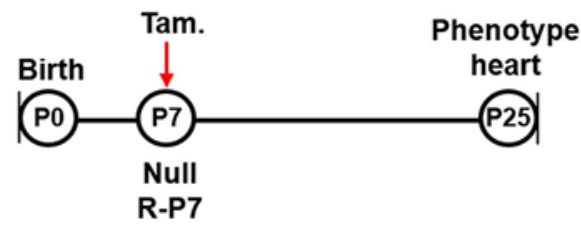

B

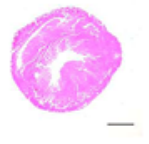

Ctl

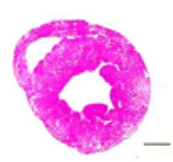

Null
C

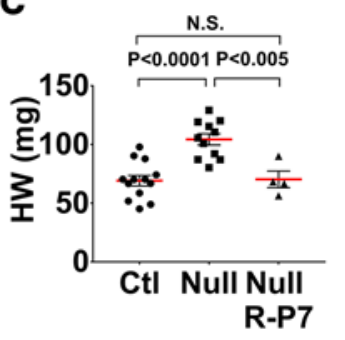

D

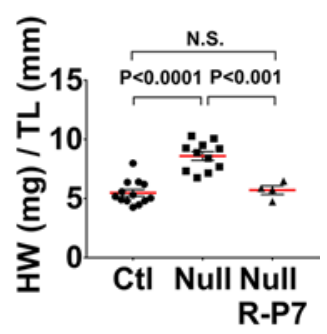

Figure 3. There is a narrow developmental period when cardiomyopathy can be reversed. (A) Schematic illustration of MYBPC3 conditional reactivation (Null R-P7) mice administered tamoxifen at P7 and phenotyped at P25. (B) H\&E-stained cross sections of control (Ctl), MYBPC3-null (Null), and Null R-P7 hearts. Scale bars: 1 $\mathrm{mm}$. (C and D) P25 heart weight (HW) (C) and HW to tibia length ratio (HW/ TL) (D) of CtI $(n=13)$, Null $(n=11)$, and Null R-P7 $(n=4)$ mice. (E) Schematic illustration of MYBPC 3 conditional reactivation (Null R-P30) mice administered tamoxifen at $\mathrm{P} 30$ and phenotyped at P60. (F) H\&E-stained cross-sections of Ctl, Null, and Null R-P30 hearts. Scale bars: $1 \mathrm{~mm}$. (G and $\mathbf{H ) ~ P 6 0 ~ H W ~ ( G ) ~ a n d ~ H W / T L ~ ( H ) ~ i n ~}$ CtI $(n=7)$, Null $(n=5)$, and Null R-P30 $(n=4)$ mice. All results are shown as mean \pm SEM. Statistical analysis performed using an unpaired, 2-tailed Student's $t$ test. N.S., not significant.

$\mathbf{F}$

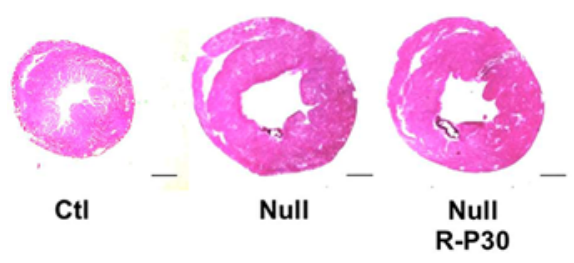

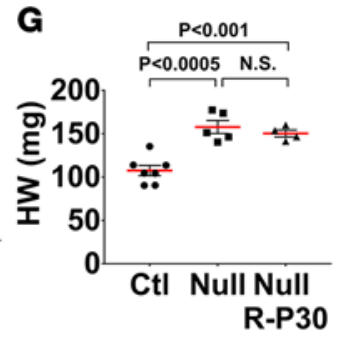

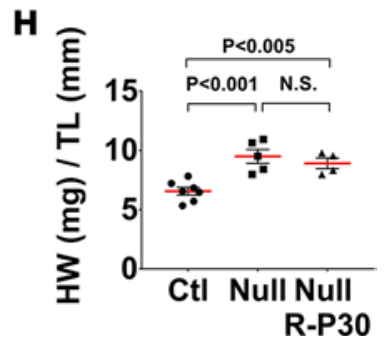

We also performed staining for nuclear pericentriolar material 1 protein (PCM-1) to selectively identify cardiomyocyte nuclei to confirm the earlier results using sarcomeric $\alpha$-actinin as a cardiomyocyte marker (13-16). First, we confirmed that PCM-1 identifies cardiomyocyte nuclei and does not stain noncardiomyocyte populations in our myocardial tissue sections (Supplemental Figure 3, D-F). We then confirmed that P7 MYBPC3-null cardiomyocytes had increased levels of DNA synthesis compared with control cardiomyocytes (Supplemental Figure 3, G and H). Finally, we used PCM-1/aurora B dual staining to measure active cardiomyocyte cytokinesis and this detected no differences between control and MYBPC3-null cardiomyocytes at P7 (Supplemental Figure 3, I and J).

We then tested if the changes we detected in cardiomyocyte DNA synthesis could be prevented through reactivation of MYBPC3 protein production at P2 (Null R-P2). Indeed, Null R-P2 animals had levels of cardiomyocyte DNA synthesis and mitosis that reverted to those of control animal levels at P25 (Figure 4, $\mathrm{F}$ and G). Once again, active cardiomyocyte cytokinesis was similar in all 3 groups of animals (Figure $4 \mathrm{H}$ ). This confirms that the changes in early postnatal heart development that we measured were initiated after birth and were not secondary to changes in embryonic heart development.

We then measured cardiomyocyte DNA synthesis in the Null C adult heart at P30. In contrast to the early postnatal period, we saw no increase in DNA synthesis in cardiomyocytes that had MYBPC3 protein expression eliminated in the adult time period (Supplemental Figure 4, A and B). This suggests that the early postnatal developmental time period is important in regulating the alterations in DNA synthesis that we detect in the MYBPC3-null cardiomyocytes.

Although aurora B kinase cleavage furrow staining is a reliable marker of cardiomyocyte cytokinesis, it is possible that cardiomyocyte number could still be altered through changes in embryonic development or through loss of cardiomyocytes through cell death. Therefore, we directly counted cardiomyocytes on LV sections from control, germline MYBPC3-null, and MYBPC3-reactivation animals. Since the LV myocardial surface area is larger in MYBPC3-null animals, we developed a method to account for differences in myocardial surface area (Supplemental Figure 4C). We detected no differences in total cardiomyocytes per 
A

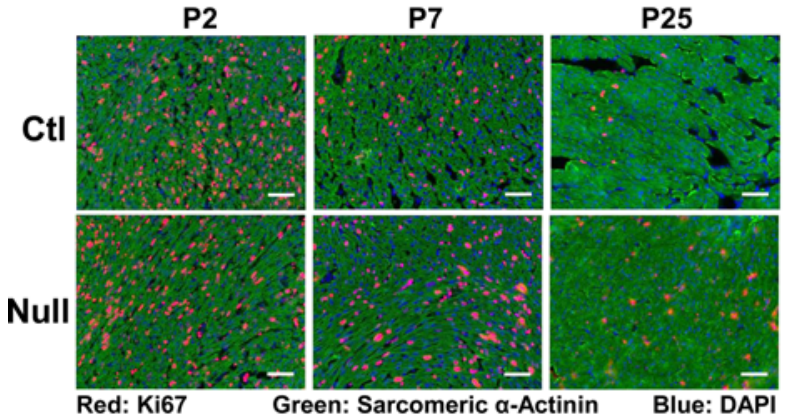

D

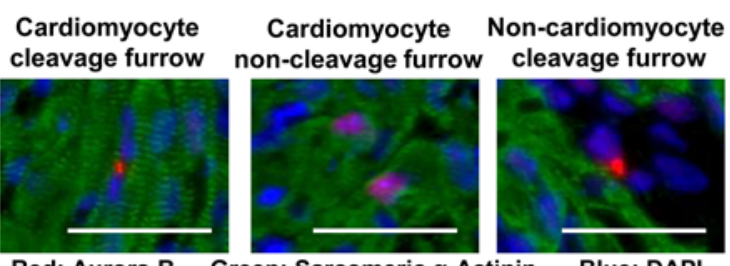

Red: Aurora B Green: Sarcomeric $\alpha$-Actinin Blue: DAPI
B
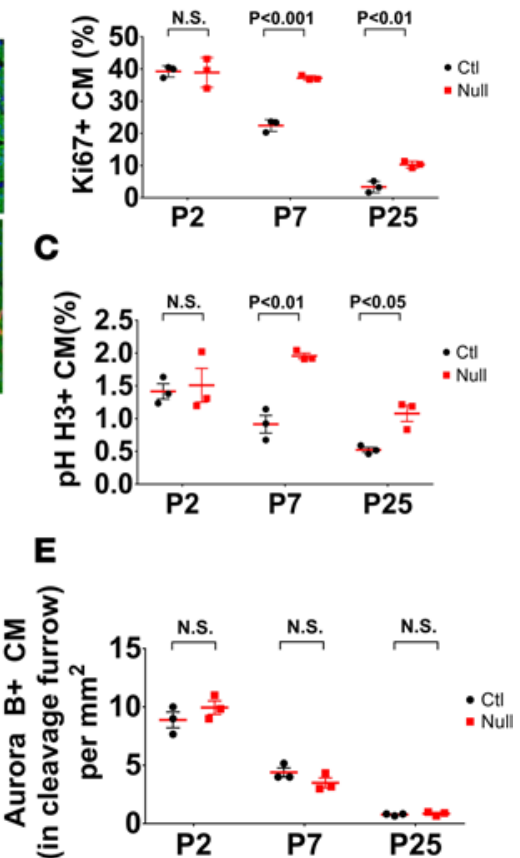

$\mathbf{F}$
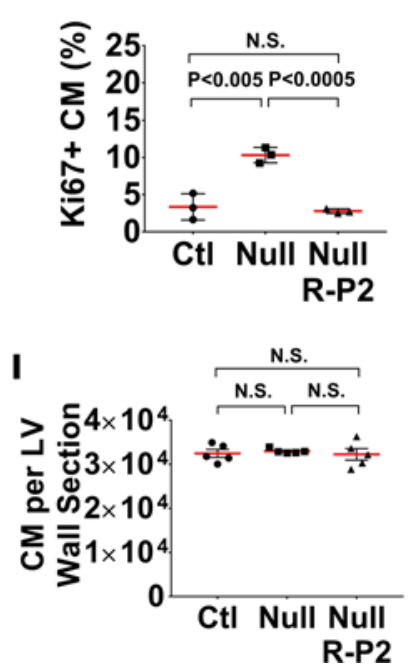

G

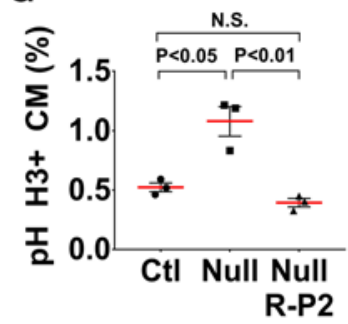

H

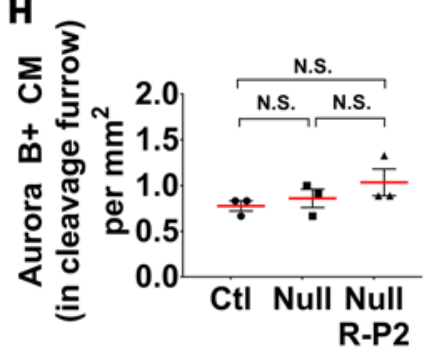

Figure 4. MYBPC3 cardiomyopathy alters the hyperplastic to hypertrophic transition phase of heart development. (A) Immunofluorescence staining of Ki67 (red) in control (Ctl) and MYBPC3-null (Null) myocardial tissue at P2, P7, and P25. Cardiomyocytes were identified with sarcomeric $\alpha$-actinin (green), with nuclei labeled with DAPI (blue). Scale bars: $100 \mu \mathrm{m}$. (B) Quantification of Ki67-positive cardiomyocytes (CMs) in CtI ( $n=3$ per age) and Null ( $n=3$ per age) hearts (minimum 4,000 cells per sample). (C) Quantification of phosphohistone $\mathrm{H3}$-positive ( $\mathrm{pH}$ H3-positive) CMs in CtI ( $n=3$ per age) and Null ( $n$ $=3$ per age) hearts (minimum 4,000 cells per sample). (D) Immunofluorescence staining of aurora $B$ (red) in myocardial tissue demonstrating aurora B staining in CM cleavage furrow (left), CM non-cleavage furrow (middle), and non-CM aurora B cleavage furrow (right). CMs were identified with sarcomeric $\alpha$-actinin (green), with nuclei labeled with DAPI (blue). Scale bars: $100 \mu \mathrm{m}$. (E) Quantification of aurora B cleavage furrow-positive CMs per $\mathrm{mm}^{2}$ in Ctl ( $n=3$ per age) and Null ( $n=3$ per age) hearts. (F-H) Quantification of Ki67 (F), $\mathrm{pH} \mathrm{H3}(\mathbf{G})$, and aurora B cleavage furrow CMs $(\mathbf{H})$ from $\mathrm{Ctl}(n=3)$, Null $(n=3)$, and MYBPC 3 conditional reactivation administered tamoxifen at P2 (Null R-P2) $(n=3)$. (I) Quantification of total CMs per left ventricular (LV) wall section in CtI $(n=5)$, Null ( $n$ $=5)$, and Null R-P2 $(n=5)$ hearts. All results are shown as mean \pm SEM. Statistical analysis performed using an unpaired, 2-tailed Student's $t$ test. N.S., not significant.

ventricular cross section comparing control, germline MYBPC3-null, and MYBPC3-reactivation animals (Figure 4I). Therefore, both our aurora B kinase cleavage furrow staining and direct cardiomyocyte counts confirm that despite increased cardiomyocyte DNA synthesis there are no changes in cardiomyocyte proliferation in MYBPC3-null animals. Therefore, the increase heart mass detected in MYBPC3-null animals was secondary to increased cardiomyocyte hypertrophy and not hyperplasia.

MYBPC3-null cardiomyocytes have increased rates of endoreplication. After detecting increased levels of DNA synthesis without evidence of cardiomyocyte proliferation, we then tested if there were changes in cardiomyocyte endoreplication. This was performed by isolating cardiomyocytes from P25 control and MYBPC3-null animals (Figure 5A). We detected a reduction in binucleated cardiomyocytes and an increase in multinucleated cardiomyocytes in the MYBPC3-null animals (Figure 5B). In addition, we demonstrate that cardiomyocyte area increases as the number of nuclei per cardiomyocyte increases, with a more pronounced effect in the MYBPC3-null cardiomyocytes (Figure 5C). These results verify that the increased cardiomyocyte DNA synthesis is contributing to increased cardiomyocyte endoreplication in the MYBPC3-null animals. 
A
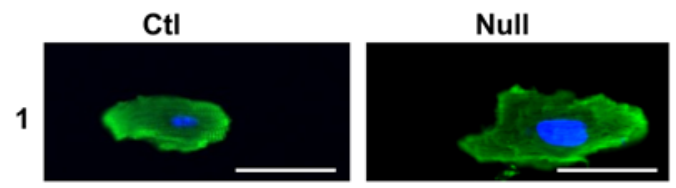

2
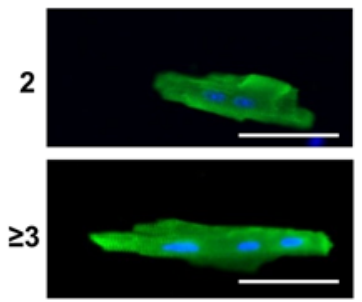

Green: Sarcomeric $\alpha$-Actinin
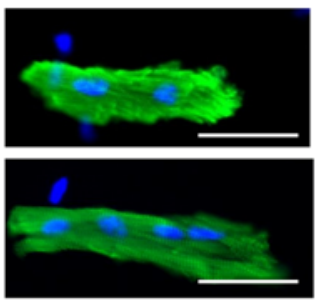

Blue: DAPI
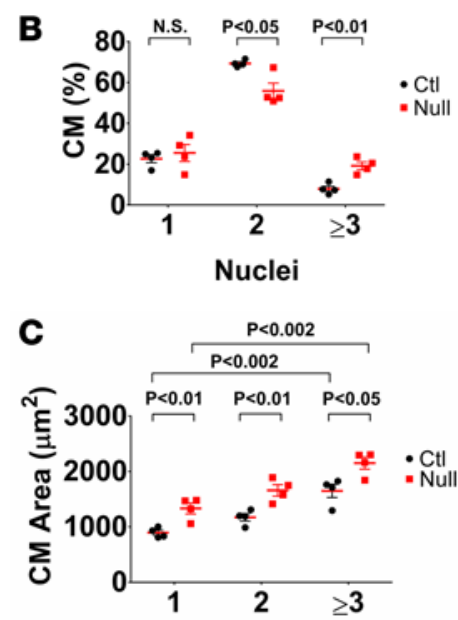

$\mathbf{F}$
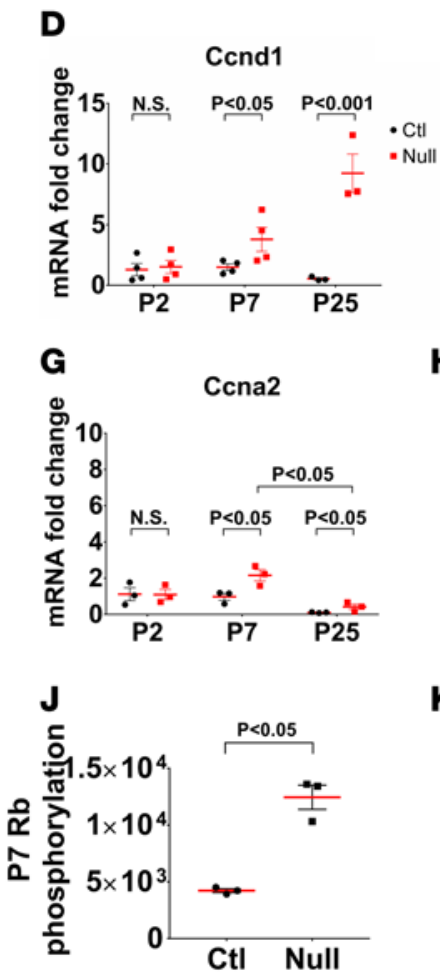

E

\section{H}

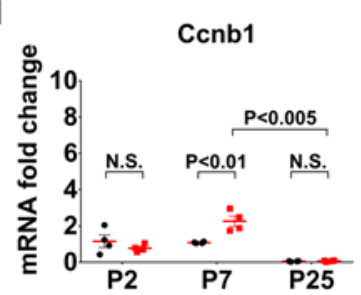

K

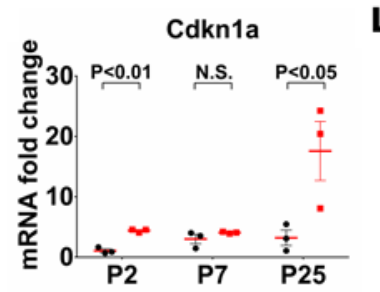

Ccnd2

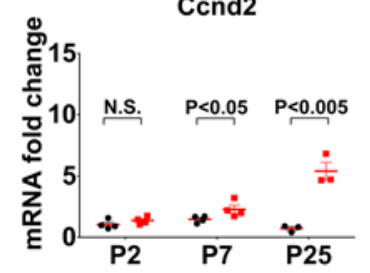

(1)

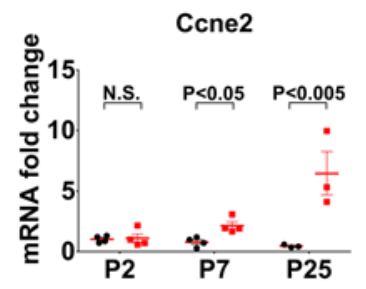

I

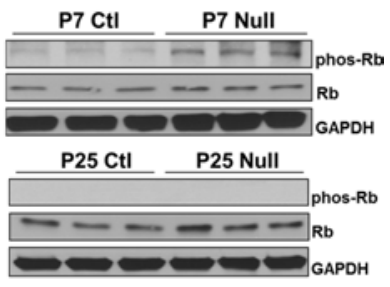

$\mathbf{L}$

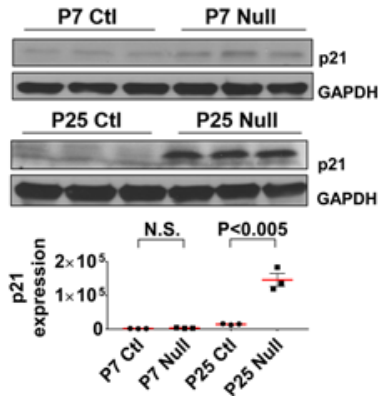

Figure 5. Dysregulated cell cycle regulation results in increased cardiomyocyte endoreplication in MYBPC3-null hearts. (A) Isolated cardiomyocytes (CMs) from 25-day-old control (CtI) and MYBPC3-null (Null) hearts displaying mononucleated (1), binucleated (2), and multinucleated $(\geq 3)$ CMs. CMs were stained with sarcomeric $\alpha$-actinin (green), with nuclei labeled with DAPI (blue). Scale bars: $50 \mu \mathrm{m}$. (B and C) Quantification of P25 CM nucleation (B) and cell size (C) in isolated CMs from CtI $(n=4)$ and Null $(n=4)$ hearts. A minimum of 300 CMs per heart were analyzed. CM nucleation represented as percentage of total CMs counted. (D-H) Measurement of gene expression of cyclin D1 (Ccnd1) (D), cyclin D2 (Ccnd2) (E), cyclin E2 (Ccne2) (F), cyclin A2 (Ccna2) (C), and cyclin B1 (Ccnb1) (H) at P2, P7, and P25 from CtI ( $n=3-4)$ and Null ( $n$ $=3-4$ ) hearts. The genes of interest were normalized to Rpl32 expression. Fold changes are shown relative to $\mathrm{P} 2$ CtI gene expression. (I) Western blot of phospho-retinoblastoma (phos-Rb) and total $\mathrm{Rb}$ protein levels from $\mathrm{P} 7$ and P25 Ctl and Null hearts. (J) Relative quantification of phos-Rb in Ctl $(n=3)$ and Null $(n=3)$ P7 hearts normalized to GAPDH. (K) Gene expression for p21 (Cdkn1a) at P2, P7, P25 from CtI $(n=3)$ and Null $(n=3)$ hearts. These data were normalized to Rpl32 expression and fold changes are shown relative to $\mathrm{P} 2 \mathrm{Ctl}$ gene expression. (L) Western blot of p21 protein levels in Ctl and Null P7 and P25 hearts (top). Relative quantification of p21 in CtI $(n=3)$ and Null $(n=3)$ P7 and P25 hearts normalized to CAPDH. All results are shown as mean \pm SEM. Statistical analysis performed using an unpaired, 2-tailed Student's $t$ test. N.S., not significant.

MYBPC3-null animals have alterations in cell cycle regulatory pathways. The major regulators of cellular DNA synthesis are the cyclin proteins, which regulate the progression of the cell cycle. We discovered that the $G_{1}$ phase regulator $(\mathrm{C} c n d 1 / 2)$ and $G_{1} / S$ regulator $(C c n e 2)$ were induced in MYBPC3-null myocardial tissue at P7 and more strongly induced at P25 (Figure 5, D-F). In addition, we found that the S and $G_{2}$ phase regulator (Ccna2), and $M$ phase regulator (Ccnb1) were induced at $P 7$ (Figure $5, G$ and $H$ ). However, in contrast to $\mathrm{C} c n d 1 / 2$ and $\mathrm{Ccne} 2$, the $\mathrm{S} / \mathrm{G}_{2} / \mathrm{M}$ phase cyclins (Ccna2 and Ccnb1) significantly decreased from P7 to P25 in control and MYBPC3-null animals (Figure 5, G and H). Additional cyclin isoforms were measured (Ccne1, Ccna1, Ccnb2, and Ccnb3), but their expression was minimal in control and MYBPC3-null myocardial tissue.

The E2F transcription factors are the major regulator of Ccna2 and Ccnb1 gene expression and the activity of these transcription factors is repressed by binding of retinoblastoma protein $(\mathrm{Rb})$. However, when $\mathrm{Rb}$ is phosphorylated, it is released from E2F transcription factors and this allows transcription of Ccna and Ccnb gene isoforms to proceed. We measured phosphorylation of $\mathrm{Rb}$ at $\mathrm{P} 7$ and discovered a greater than 2-fold increase in phospho- $\mathrm{Rb}$ in MYBPC3-null animals compared with control hearts 
(Figure 5, I and J). In contrast, by P25 there was no detectable phospho-Rb in control or MYBPC3-null myocardial tissue (Figure 5I).

Despite robust expression of Ccnd1/2 and Ccne2 in the MYBPC3-null hearts at P25, there was no detectable phospho- $\mathrm{Rb}$. Therefore, we measured the expression of the cyclin-dependent kinase inhibitor p21 (Cdkn1a) and discovered a strong induction of p21 gene and protein expression between P7 and P25 in MYBPC3-null animals (Figure 5, K and L). Collectively, these results suggest that dysregulated cell cycle activity contributes to the process of enhanced cardiomyocyte endoreplication in early postnatal cardiomyocytes deficient in MYBPC3.

Reduction of cardiomyocyte endoreplication modifies the development of cardiomyopathy in MYBPC3-null animals. Next, we wanted to determine if manipulation of cardiomyocyte endoreplication would modify the development of cardiomyopathy in the MYBPC3-null animals. Therefore, we utilized the CDK4/6 inhibitor PD-0332991 to inhibit cyclin D-CDK4/6 activity during the P2 to P7 time interval (Figure 6A). We found that increasing concentrations of PD-0332991 were effective in reducing cardiomyocyte DNA synthesis during this time period, with a more robust effect in the MYBPC3-null cardiomyocytes compared with controls (Figure 6, B and C). We then tested if cardiomyocyte endoreplication was altered by reducing cardiomyocyte DNA synthesis. Indeed, we found that there was a reduction in both cardiomyocyte binucleation and multinucleation secondary to CDK4/6 inhibition (Figure 6, D-F). Both control and MYBPC3-null cardiomyocytes shifted from majority binucleated to majority mononucleated cardiomyocyte populations. Despite the reduction in cardiomyocyte DNA synthesis and endoreplication, we did not detect any evidence of increased cardiomyocyte apoptosis as assessed by TUNEL staining (Supplemental Figure 5, A and B) or reduction in total cardiomyocyte number (Supplemental Figure 5, C and D) following CDK4/6 inhibition.

Next, we wanted to determine if a reduction in cardiomyocyte endoreplication altered the development of cardiomyopathy in the MYBPC3-null animals. We found that the increased cellular hypertrophy seen in the MYBPC3-null cardiomyocytes was prevented by CDK4/6 inhibition (Figure 6G). Likewise, the increased cardiac mass in the MYBPC3-null animals was reduced to almost control levels by CDK4/6 inhibition (Figure $6 \mathrm{H}$ and Supplemental Figure 5E). In contrast to cardiac mass, CDK4/6 inhibition had a minimal effect on body weight in control mice and no effect in MYBPC3-null mice (Supplemental Figure $5 F)$. Importantly, cardiac systolic function was also significantly improved in the MYBPC3-null animals exposed to PD-0332991 (Figure 6I and Supplemental Figure 6A). A reduction in myocardial wall thickness and LV dilation in the MYBPC3-null animals exposed to the CDK4/6 inhibitor was also observed (Supplemental Figure 6, B-D). Overall, these data show that MYBPC3 cardiomyopathy causes dysregulated cell cycle activity during early postnatal heart development, which leads to increased cardiomyocyte endoreplication and progression of cardiomyopathy (Figure 7).

\section{Discussion}

Although sarcomere gene mutations are expressed throughout cardiogenesis, most carriers of these mutations develop evidence of cardiomyopathy well after birth. Murine models with altered sarcomere function have highlighted the importance of the early postnatal period in the pathogenesis of sarcomeric cardiomyopathy $(8-11,17)$. However, it has remained unclear why this developmental period is important in the pathogenesis of disease. Using transgenic murine models that allow conditional deletion and reactivation of the sarcomere protein MYBPC3, we discovered alterations in the hyperplastic to hypertrophic transition phase of postnatal heart development that lead to increased cardiomyocyte endoreplication. We found that there was increased expression of the $G_{1} / S$ phase cyclin genes (Ccnd1/2 and Ccne2) at P7, which led to increased phosphorylation of $\mathrm{Rb}$ and subsequent expression of the $\mathrm{G}_{2} / \mathrm{M}$ phase cyclins (Ccna2 and Ccnb1). However, despite persistent high-level expression of the $\mathrm{G}_{1} / \mathrm{S}$ phase cyclin proteins at P25, cardiomyocyte DNA synthesis was greatly diminished in MYBPC3-null animals. The mechanism for this reduction in cell cycle activity at P25 in the MYBPC3-null animals was a profound upregulation of the cyclin kinase inhibitor p21. Interestingly, inhibition of cardiomyocyte endoreplication in MYBPC3-null animals altered the development of sarcomeric cardiomyopathy during the early postnatal time period. This experimental work provides valuable insights into how alterations in sarcomere protein function modify postnatal heart development and highlight the importance of developmental pathways in modifying the pathogenesis of cardiomyopathic stimuli.

During early postnatal mammalian heart development, cardiomyocytes transition from a state of hyperplastic to hypertrophic growth (12). During this transition to hypertrophic growth, there is a period where cardiomyocyte DNA synthesis persists while cytokinesis is prevented, which creates multinucleated and/or 
A

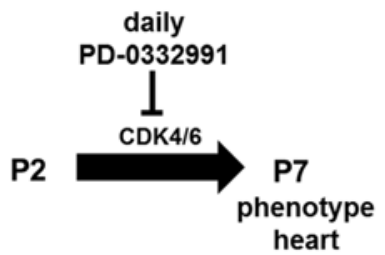

C

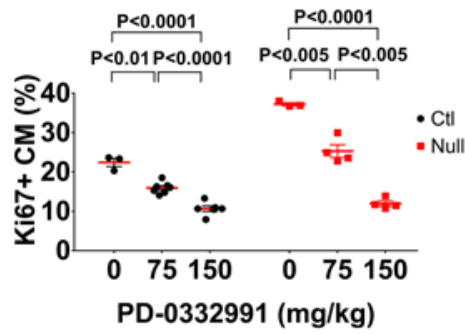

E

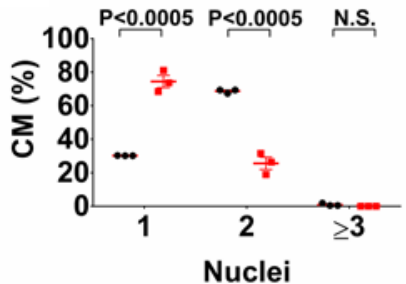

G

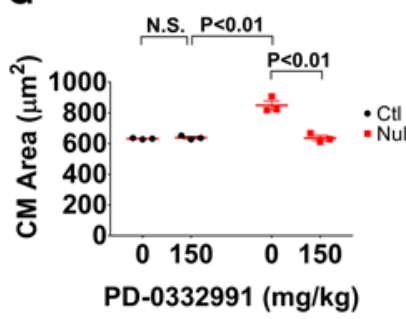

H
B
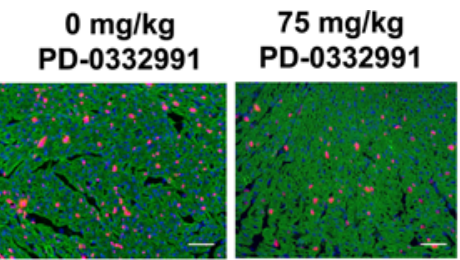

$150 \mathrm{mg} / \mathrm{kg}$

PD-0332991

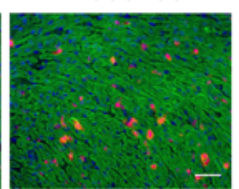

Null
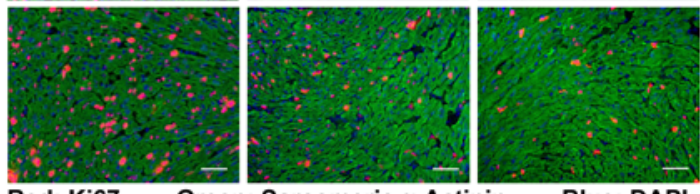

D

$0 \mathrm{mg} / \mathrm{kg}$ PD-0332991

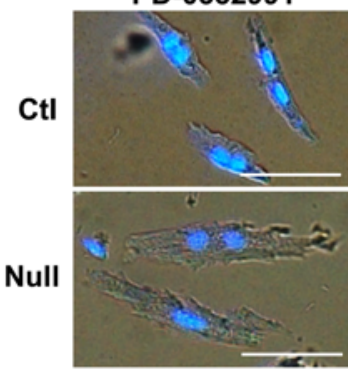

Blue: DAPI

$\mathbf{F}$
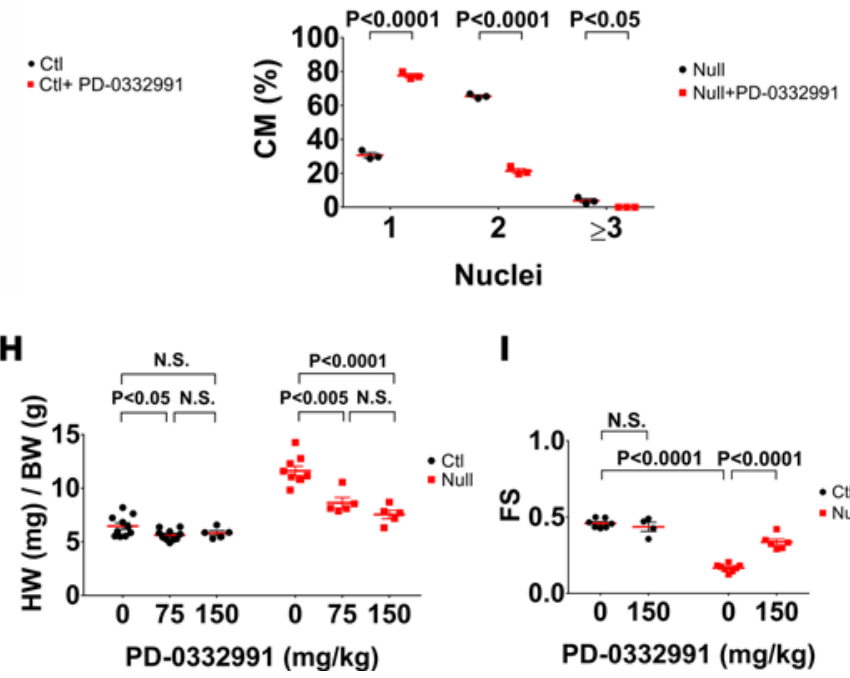

I

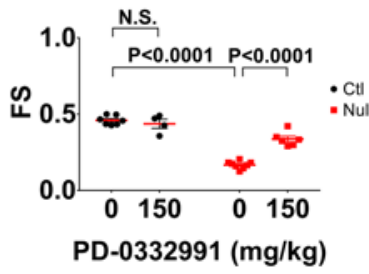

Figure 6. Reduction of cardiomyocyte endoreplication alters cardiomyopathy development in MYBPC3-null animals. (A) Schematic illustration of cell cycle inhibition from P2 to P7 with the CDK4/6 inhibitor PD-0332991. (B) Measurement of cardiomyocyte (CM) Ki67 (red) expression in control (CtI) and MYBPC3-null (Null) hearts at P7 from mice administered 75 or $150 \mathrm{mg} / \mathrm{kg}$ PD-0332991. CMs were identified with sarcomeric $\alpha$-actinin (green), with nuclei labeled with DAPI (blue). Scale bars: $100 \mu \mathrm{m}$. (C) Quantification of Ki67-positive CMs in CtI ( $n=3), \mathrm{Ctl}+75 \mathrm{mg} / \mathrm{kg}$ PD-0332991 ( $n=7), \mathrm{Ctl}+150 \mathrm{mg} / \mathrm{kg}$ PD-0332991 ( $n=6$ ), Null $(n=3)$, Null $+75 \mathrm{mg} / \mathrm{kg}$ PD-0332991 $(n=4)$, and Null $+150 \mathrm{mg} / \mathrm{kg} \mathrm{PD-0332991}(n=4)$ hearts (minimum 4,000 cells per sample). (D) Isolated CMs from P7 Ctl and Null mice administered vehicle or $150 \mathrm{mg} / \mathrm{kg}$ PD-0332991. CM nuclei were labeled with DAPI (blue). Scale bars: $50 \mu \mathrm{m}$. (E) Quantification of CM nucleation at P7 from CtI $(n=3)$ mice exposed to vehicle or $150 \mathrm{mg} / \mathrm{kg}$ PD-0332991. A minimum of $300 \mathrm{CMs}$ per heart were analyzed. Results represented as percentage of total CMs counted. (F) Quantification of CM nucleation at P7 from Null $(n=3)$ mice exposed to vehicle or $150 \mathrm{mg} / \mathrm{kg}$ PD-0332991 (minimum of 300 cardiomyocytes per sample). (C) Quantification of isolated CM area from CtI $(n=3)$ and Null $(n=3)$ P7 mice exposed to vehicle or $150 \mathrm{mg} / \mathrm{kg}$ PD-0332991 (minimum of 300 cardiomyocytes per sample).

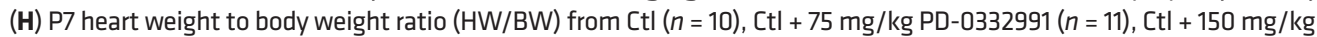
PD-0332991 ( $n=5)$, Null $(n=9)$, Null $+75 \mathrm{mg} / \mathrm{kg}$ PD-0332991 ( $n=5)$, and Null $+150 \mathrm{mg} / \mathrm{kg} \mathrm{PD-0332991} \mathrm{(} n=5)$ mice. (I) P7 fractional shortening (FS) obtained from M-mode echocardiography of Ctl $(n=7), \mathrm{Ctl}+150 \mathrm{mg} / \mathrm{kg}$ PD-0332991 $(n=4)$, Null $(n=8)$, and Null $+150 \mathrm{mg} / \mathrm{kg}$ PD-0332991 $(n=6)$ mice. All results are shown as mean \pm SEM. Statistical analysis performed using an unpaired, 2-tailed Student's $t$ test. N.S., not significant. 
A

P7 MYBPC3 Null

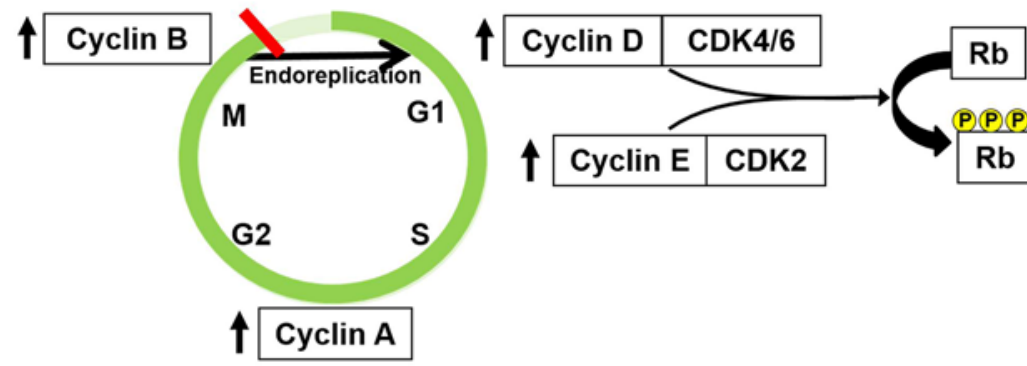

B

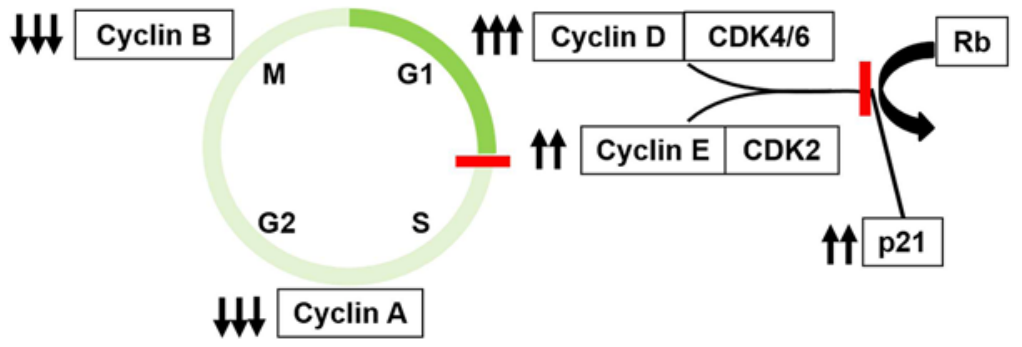

Figure 7. Mechanistic overview of cell cycle alterations in MYBPC3-null hearts at P7 and P25. (A) MYBPC3-null hearts at P7 (P7 MYBPC3 Null) have increased expression of cyclin $\mathrm{D}$ and $\mathrm{E}$, which results in increased phosphorylation of Rb protein. Enhanced phosphorylation of Rb protein reduces the transcriptional repression of cyclin $A$ and $B$ gene expression. With increased expression of key cell cycle cyclins, the cell cycle advances, causing increased cardiomyocyte DNA synthesis without cardiomyocyte cell division (endoreplication). (B) At P25, MYBPC3-null hearts still have significantly elevated levels of cyclin $D$ and $E$. However, there is elevated expression of p21 protein, which reduces the phosphorylation of Rb by the cyclin D/CDK4 complex. This allows nonphosphorylated $\mathrm{Rb}$ to maintain its role as a repressor of cyclin $A$ and $B$ gene transcription. Therefore, cyclin A and B expression is low at this time point and cardiomyocyte DNA synthesis is significantly reduced compared with P7 MYBPC3 Null cardiomyocytes.

polyploid cardiomyocytes (a form of endoreplication) (18). This developmental process has been intensively studied in an effort to understand molecular mechanisms regulating cardiomyocyte proliferation. Changes in transcription factor regulation, miRNA expression, hormone levels, and oxidative DNA damage have been shown to be important in controlling this

developmental phenomenon (19-22). Our data demonstrate that the hyperplastic to hypertrophic transition phase of postnatal heart development is modified by sarcomere dysfunction and that this dysregulated cell cycle activity contributes to the pathogenesis of disease. Interestingly, an upregulation of p21 appears to serve as a counter-regulatory mechanism employed by cardiomyocytes during this developmental stage to prevent persistent cardiomyocyte DNA synthesis in the setting of pathogenic stimuli. High levels of p21 have been demonstrated to inhibit the activity of cyclin D-CDK4/6 and cyclin E-CDK2 and, therefore, prevent cell cycle progression $(23,24)$.

Endoreplication occurs in both vertebrate and invertebrate organisms during normal development and secondary to pathogenic stimuli (25-27). Endoreplication has been shown to play a role in the regulation of cell size during organogenesis (28). In addition to regulating developmental growth, endoreplication is necessary for normal function of various mammalian cell types. For example, platelet-precursor megakaryocytes undergo endoreplication to increase cell size - a process necessary for appropriate maturation and fragmentation to form platelets (29). Trophoblast giant cells also utilize rapid endoreplication for proper differentiation and formation of the outer epithelial lining of the placenta (30). Previous studies have suggested a role for endoreplication in cellular hypertrophy as both Drosophila epithelium and mammalian hepatocytes utilize endoreplication to increase cell size rather than cell number in order to compensate for tissue damage $(31,32)$. Interestingly, elevated levels of DNA synthesis and DNA damage have been shown to be reciprocally associated with endoreplication in other cell types $(25,33)$. Future work will be necessary to determine the role of DNA damage-response pathways in regulating cardiomyocyte endoreplication in the setting of development and disease.

The role of endoreplication in the developing cardiomyocyte is less clear; however, it has been suggested that the ability to generate an abundance of genetic template for protein synthesis would be advantageous in a cardiomyocyte undergoing hypertrophy associated with normal heart growth (18). Furthermore, the induction of DNA synthesis in the adult heart caused cardiomyocyte endoreplication and hypertrophy to develop (34). We discovered that cardiomyocyte endoreplication was enhanced in the setting of sarcomere protein dysfunction during the early postnatal time period. However, it remained unclear if the increased endoreplication was a protective or pathological response in the MYBPC3-null cardiomyocytes. Therefore, we inhibited the $\mathrm{G}_{1}$ phase of the cell cycle by administering the highly selective CDK4/6 inhibitor PD-0332991 during the earliest phase of pathological heart growth. We found that both control and MYBPC3-null hearts shifted from predominantly binucleated to mononucleated cardiomyocytes, which confirmed that our strategy was successful in preventing endoreplication. Interestingly, the reduction in cardiac mass was much greater in the MYBPC3-null animals compared with control animals. In addition, 
cardiac systolic function improved in the MYBPC3-null animals treated with PD-0332991 and control animals had no significant change in systolic function. Importantly, we saw no evidence of increased cardiomyocyte apoptosis in either control or MYBPC3-null animals treated with the CDK4/6 inhibitor. These results suggest that cardiomyocyte endoreplication is contributing to the pathogenesis of cardiomyopathy in the MYBPC3-null animals.

We found that upregulation of the cyclin kinase inhibitor p21 in MYBPC3-null animals coincides with a reduction in $\mathrm{Rb}$ phosphorylation and reduction in cardiomyocyte cell cycle activity by $\mathrm{P} 25$. This suggests that an endogenous process is initiated in the MYBPC3-null animals to prevent persistent activation of the cardiomyocyte cell cycle at later stages of development. Studies in which the Myh6 promoter was used to drive expression of cyclin D1, D2, or A2 in the mouse heart showed significant increases in cardiomyocyte DNA synthesis, which support our finding that upregulation of cyclins can increase cardiomyocyte DNA synthesis (35-37). However, Myh6:cyclinA2 transgenic animals had a rapid decrease in both proliferating cellular nuclear antigen (PCNA) and phospho-histone H3 by P14, despite persistent high-level expression of cyclin A2. Likewise, despite constitutive expression of cyclin D1 in the Myh6:cyclinD1 transgenic animals, only 0.04\% of adult cardiomyocytes showed evidence of active DNA synthesis. In a different study, cyclin D1 was induced in adult cardiomyocytes, which increased cardiomyocyte DNA synthesis, but again this effect was only transient and diminished rapidly by 14 days after induction (38). The results from these studies support the conclusion that endogenous mechanisms are initiated to reduce persistent activation of the cardiomyocyte cell cycle in adult mammalian cardiomyocytes. Our data suggest that upregulation of p21 plays a role in this process. However, data from conditional cyclin D1 overexpression in adult cardiomyocytes suggest there are also p21-independent mechanisms. One potential mechanism is that persistent overexpression of cyclin D1 might interfere with the normal nuclear export of cyclin $D$ that is required for $G_{1}$ to $S$ phase transition $(39,40)$. In addition, it is possible that healthy and diseased cardiomyocytes utilize different pathways to regulate postnatal DNA synthesis.

Despite the robust cardiomyocyte DNA synthesis detected in the MYBPC3-null animals, we did not detect evidence of increased cardiomyocyte proliferation. In contrast, we detected increased cardiomyocyte endoreplication and cardiomyocyte hypertrophy in MYBPC3-null cardiomyocytes. Interestingly, a murine model expressing a truncated MYBPC3 protein was reported to have increased cardiomyocyte proliferation in homozygous animals during the early postnatal time period (17). One explanation for the divergent results between our data and those of Jiang et al. may be the different methods utilized to manipulate MYBPC3 protein expression. Our models have no detectable MYBPC3 protein expression in the homozygous state, whereas the MYBPC3 truncation model utilized by Jiang et al. does still express low levels of MYBPC3 protein in the homozygous state (41). It is possible that the low level of truncated MYBPC3 protein expression has a unique biological role that is not replicated in our models which are null for MYBPC3 protein. Another potential reason for the divergent results between the 2 models is the genetic background of the mouse strains. The MYBPC3-truncation mouse model was in a $129 \mathrm{SvEv}$ genetic background and our MYBPC3 models were bred in a C57BL/6 genetic background. Different inbred mouse strains have been reported to have unique cardiovascular responses to pathological stimuli $(42,43)$. Likewise, different inbred mouse strains have been shown to have divergent regenerative capabilities (e.g., the MRL line), suggesting cell cycle regulation may be modified by genetic background (44). In addition, it was reported that neuronal tissue from the $129 \mathrm{SvJ}$ inbred strain has increased proliferative potential compared with neuronal tissue from the C57BL/6 strain (45). Another potential explanation for the divergent results in the 2 MYBPC3 models is the experimental methods utilized. Both groups performed aurora B kinase staining to detect active cardiomyocyte cytokinesis in P7 myocardial tissue. We utilized both sarcomeric $\alpha$-actinin/aurora B and PCM-1/aurora B immunohistochemistry to identify 2.5 to 4.4 cleavage furrow-positive cardiomyocytes per $\mathrm{mm}^{2}$ in control and MYBPC3-null myocardial tissue at P7. In contrast, Jiang et al. reported that control myocardial tissue had fewer than 0.5 cleavage furrow-positive cardiomyocytes per $\mathrm{mm}^{2}$ at $\mathrm{P}$, while the MYBPC3-truncation homozygous animals had $\sim 4$ cleavage furrow-positive cardiomyocytes per $\mathrm{mm}^{2}$. Other groups have reported aurora B cardiomyocyte cleavage furrow values that range from $\sim 1.75$ to 5 per field or $\mathrm{mm}^{2}$ at the $\mathrm{P} 7$ to $\mathrm{P} 8$ time point. Therefore, it would appear that the aurora B cleavage furrow values reported by Jiang et al. for control myocardial tissue at P7 are low. In addition, Jiang et al. did not adequately account for the role of cardiomyocyte hypertrophy in contributing to the increase in heart mass in their MYBPC3 truncation model. 
We measured a $46 \%$ increase in cardiomyocyte cross-sectional area and a $38 \%$ increase in total cardiomyocyte area in MYBPC3-null cardiomyocytes at P25. This significant increase in cardiomyocyte hypertrophy is the primary reason for the increased heart mass we measured at P25 in this MYBPC3-null model. Finally, we also performed PCM-1 nuclear staining as an additional method to confirm our original results using sarcomeric $\alpha$-actinin staining (13-16). Using this method, we again observed no differences between control and MYBPC3-null myocardial tissue with regards to cardiomyocyte cytokinesis at P7, despite clear differences in cardiomyocyte DNA synthesis.

We identified a striking difference between the early postnatal heart and the adult heart to ablation or reactivation of MYBPC3 protein expression. When MYBPC3 protein expression was eliminated in adult cardiomyocytes, we did not detect changes in cardiac mass or fractional shortening after 60 days and these results are quite similar to an earlier report that performed conditional deletion of MYBPC3 in adult cardiomyocytes and performed initial analysis at 8 weeks after tamoxifen injection (46). Unlike the earlier study, we did not subject our mice to extended aging, exercise, or transverse aortic constriction since our goal was to directly compare the cardiac responses of the early postnatal heart and adult heart to ablation or reactivation of MYBPC3 protein expression over similar time periods. Similar to the results from the adult MYBPC3 conditional-deletion model, we also detected no significant response of the adult MYBPC3-null heart to reactivation of MYBPC3 expression. There have been multiple groups that have also demonstrated that reversal of established sarcomeric cardiomyopathy in the adult animal was not successful $(8,9,11)$. In contrast, one study used a viral strategy to express MYBPC3 protein in the adult MYBPC3-null heart and reported partial improvement in systolic function and a reduction in heart mass 21 days after viral injection (47). However, the mice in this study were injected with a MYBPC3-expressing viral vector at a range of ages spanning 8 to 26 weeks and were then analyzed together. In contrast, our study and the other studies that failed to reverse adult sarcomeric cardiomyopathy typically selected a specific adult age for their experimental groups. Longer term studies and reactivation of MYBPC3 expression at different adult ages will be required to better understand these differences.

In conclusion, we used multiple complimentary transgenic murine models to show that dysregulation of the cardiomyocyte cell cycle during the hyperplastic to hypertrophic transition phase of postnatal heart development contributes to sarcomeric cardiomyopathy. Importantly, this leads to increased cardiomyocyte endoreplication without evidence of increased cardiomyocyte proliferation. We discovered an upregulation of the cell cycle-activating cyclins during this phase of development with a corresponding increase in $\mathrm{Rb}$ phosphorylation. However, a robust upregulation of the cyclin kinase inhibitor p21 caused an inhibition of cell cycle activity at later stages of development. Importantly, inhibition of cardiomyocyte cell cycle activity and endoreplication in the MYBPC3-null animals altered the development of cardiomyopathy. This experimental work provides valuable insights into how alterations in sarcomere protein function modify postnatal heart development and highlight the potential of targeting cell cycle regulatory pathways to modify cardiomyopathy development.

\section{Methods}

Study design. The objective of this study was to determine the mechanisms underlying the development of sarcomeric cardiomyopathy in the early postnatal murine heart. All murine models were maintained in a C57BL/ 6 genetic background and both male and female mice were analyzed. Investigators were blinded to animal genotype and/or treatment at time of data collection. Minimum sample size required to achieve significance was calculated using $G^{*}$ Power 3 software (48), with $\alpha=0.05$ and $\beta=0.80$. A minimum of 3 biological replicates for all experiments was used and no animals were excluded from analysis. For all drug treatments, a group of control mice were administered vehicle only to confirm that the vehicle or delivery method had no significant effect on the measured phenotypes.

Transgenic animal lines. Mice with a knockout-first tm1a allele of the Mybpc3 gene (Mybpc ${ }^{\text {tmla }}$, EUCOMM 4435924) were rederived into a C57BL/6 background (49). The Mybpc ${ }^{\text {tmla }}$ line was then crossed with a ACTB:Flp deleter line to create a conditional Mybpc3 allele (Mybpc $\left.3^{\mathrm{tm} 1 \mathrm{c}}\right)$. The conditional Mybpc3 line was then crossed with a CMV:Cre line to create a germline null allele of Mybpc3 (Mybpc $3^{\text {tmld }}$ ). The conditional Mybpc3 line was also crossed with $\mathrm{Tg}($ Myh6-Cre/Esr1*)1Jmk (Jackson Laboratories, stock 005657) to allow tamoxifen-induced conditional deletion of Mybpc3 expression. Lastly, the Mybpc ${ }^{\text {tmla }}$ line was crossed with a tamoxifen-inducible Flp recombinase line (R26 $6^{\mathrm{FlpoER}}$, Jackson Laboratories, stock 019016) 
to allow tamoxifen-induced conditional deletion of the FRT-flanked lacZ/neo knockout-first cassette, which allows for reactivation of MYBPC3 expression (Supplemental Figure 1).

Drug treatments. For tamoxifen injections, tamoxifen (Sigma-Aldrich) was dissolved in warm sesame oil and injected subcutaneously or intraperitoneally at the doses outlined (Myh6-Cre/Esr1, $40 \mathrm{mg} / \mathrm{kg}$ / day once; R26 $6^{\mathrm{FlpoER}}, 12.5-200 \mathrm{mg} / \mathrm{kg} /$ day twice) $(50,51)$. For administration of the CDK $4 / 6$ inhibitor PD-0332991 (Active Biochemical Co.), the drug was dissolved in sodium lactate ( $\mathrm{pH} 4.0$ ) and administered daily via oral gavage at 75 or $150 \mathrm{mg} / \mathrm{kg}$ from P2 to P7.

Murine echocardiography and heart weight assessment. All echocardiographic studies were performed under conscious conditions without anesthetic. Transthoracic echocardiography was performed using a Vevo 2100 High-Resolution system (VisualSonics Inc.). Two-dimensional M-mode images were obtained and measured by an individual who was blinded to the genotype or grouping of mice. Parameters measured include LVPWd, IVSd, LV internal dimensions at end diastole (LVIDd), and end systole (LVIDs). LV fractional shortening was calculated using the formula (LVIDd - LVIDs)/LVIDd. Average heart rate for P2 control and MYBPC3-null mice was $359.8 \pm 16.98$ and $334.9 \pm 7.72$ beats per minute, respectively. Average heart rate for P7 control and MYBPC3-null mice was $455.0 \pm 9.65$ and $470.6 \pm 26.18$ beats per minute, respectively. Average heart rate for P25 control and MYBPC3-null mice was 601.1 \pm 29.47 and $616.3 \pm$ 27.59 beats per minute, respectively. After echocardiography, mice were anesthetized, total body weight was obtained, hearts were excised and perfused with $0.5 \mathrm{M} \mathrm{KCl}$, and heart weight was obtained. Mouse lower extremities were amputated at the mid-femur and boiled to remove tissues and joints. Once tibias were isolated and cleaned, digital calipers were used to measure length. Heart weight (mg) to body weight (g) or tibia length $(\mathrm{mm})$ ratios were then calculated.

Immunohistochemistry. For H\&E staining, hearts were excised and placed in paraffin or OCT. Hearts were sectioned in the apical to basal direction at a thickness of 5 to $10 \mu \mathrm{m}$ per section and subsequently stained with standard H\&E staining procedures.

For cell cycle markers, hearts embedded in OCT were sectioned on a cryostat (Leica) to produce 5 - $\mu \mathrm{m}$ sections followed by fixation with $4 \%$ paraformaldehyde (PFA). Following PBS washes, sections were permeabilized with $0.2 \%$ Triton X-100 with subsequent PBS washes. Sections were then incubated with 1\% BSA in PBS for 1 hour at room temperature. Dual primary antibody incubation with Ki67 (Cell Signaling Technology, catalog 9129), phospho-histone H3 (Cell Signaling Technology, catalog 3377), PCM-1 (Sigma-Aldrich, catalog HPA023370), isolectin B4 (Vector Laboratories, catalog DL-1207), aurora B (Abcam, catalog ab2254), or sarcomeric $\alpha$-actinin (Abcam, catalog ab9465) was carried out at $4^{\circ} \mathrm{C}$ overnight. Sections were washed with PBS and then incubated with fluorescent secondary goat antirabbit Alexa Fluor 594 (Thermo Fisher Scientific, catalog A-11005) and goat anti-mouse Alexa Fluor 488 (Thermo Fisher Scientific, catalog A-10667) antibodies for 1 hour in the dark at room temperature. When primary antibodies from similar species were utilized, antibodies underwent direct conjugation with differing fluorophores as carried out by Zenon Antibody Labeling Kits (Thermo Fisher Scientific). After PBS washes, sections were counterstained and mounted with ProLong Gold Antifade mountant with DAPI (Thermo Fisher Scientific) and allowed to cure overnight in the dark at room temperature. Slides were imaged at $\times 20$ magnification on an inverted wide-field fluorescence microscope (Olympus). Images containing DAPI were processed in ImageJ (http://imagej.nih.gov/ij/) to count total nuclei utilizing the watershed function to delineate touching nuclei. Cell cycle markers were analyzed in a similar manner and validated by manual counting.

$\beta$-Gal tissue staining was performed as per manufacturer's instructions (Thermo Fisher Scientific, catalog K1465-01), with slight modification. Tissue sections were thawed and subsequently fixed with $2 \%$ formaldehyde/0.2\% glutaraldehyde. Following PBS washes, tissue sections were incubated with staining solution (4 mM potassium ferricyanide, $4 \mathrm{mM}$ potassium ferrocyanide, $2 \mathrm{mM}$ magnesium chloride, $1 \mathrm{mg} /$ $\mathrm{ml} \mathrm{X}$-gal) overnight at $37^{\circ} \mathrm{C}$. After incubation, tissue sections were washed with PBS and mounted with ProLong Gold Antifade mountant. Slides were visualized using bright-field microscopy and $\beta$-gal-positive and -negative cardiomyocytes were quantified.

Identification of apoptotic cardiomyocytes was carried out using a VitroView TUNEL In Situ Apoptosis Detection Kit (GeneCopoeia, VB-4005R) as per manufacturer's instructions. Briefly, frozen tissue sections were thawed and fixed with $4 \%$ PFA. Following washing, sections were exposed to terminal deoxynucleotidyl transferase reagent for 30 minutes at $37^{\circ} \mathrm{C}$. Following termination of the reaction with sodium chloride and citrate buffer, slides were washed, blocked with a $2 \%$ BSA solution, and incubated 
with streptavidin-Andy Fluor 594 for 30 minutes at room temperature. Following mounting of slides with DAPI-containing mountant, TUNEL-positive cardiomyocytes were imaged with a wide-field fluorescence microscope (Olympus) and quantified in ImageJ.

Cardiomyocyte size and number. Fresh-frozen 10- $\mu \mathrm{m}$ sections were fixed for 10 minutes in 4\% PFA, followed by a wash in $1 \times$ HBSS. Slides were then incubated in the dark for 10 minutes with Oregon Green 488-conjugated wheat-germ agglutinin (WGA) (Thermo Fisher Scientific, W6748) diluted 1:20 in HBSS. Slides were rinsed in HBSS and mounted with ProlongGold Antifade. Images for cardiomyocyte size measurements were taken at $\times 40$ magnification. Four to 5 images were taken for LV septum and LV free-wall regions, in which most cells were in cross section. These images were then imported into ImageJ and only those cardiomyocytes in cross section were traced using the ROI Manager function to find the average cardiomyocyte cross-sectional area for each region.

In order to determine cardiomyocyte number, WGA images were taken from endocardium to epicardium in 4 distinct LV myocardial regions: septum, anterior, lateral, and inferior wall. Each cardiomyocyte was manually counted using ImageJ's Cell Counter function. Cardiomyocytes were defined as cells with an uninterrupted border of WGA stain and a dark cell center. An average of 12,000 cardiomyocytes were counted per biological replicate. Once an image was analyzed, its area was measured to give a cardiomyocyte number per area (cardiomyocytes $/ \mathrm{mm}^{2}$ ) and the average of the 4 regions was calculated (average cardiomyocytes $/ \mathrm{mm}^{2}$ ). To account for differences in total LV myocardial surface area, images of the entire LV myocardium were taken on a dissecting microscope and the entire LV was traced in ImageJ to calculate the total LV myocardial surface area in $\mathrm{mm}^{2}$. This LV surface area was then multiplied by the average cardiomyocytes $/ \mathrm{mm}^{2}$ to yield the total cardiomyocyte number for each LV cross section (Supplemental Figure 4C).

Cardiomyocyte nucleation. Cardiomyocytes from P7 or P25 hearts were isolated as previously described with slight modification (52). Briefly, hearts were minced into small pieces with subsequent fixation with $4 \%$ PFA for 4 hours at $4^{\circ} \mathrm{C}$. Fixed tissue was enzymatically digested with $0.1 \mathrm{mg} / \mathrm{ml}$ Liberase DH (Sigma-Aldrich, cata$\log$ number 5401054001) with shaking at $37^{\circ} \mathrm{C}$ until fully dissociated. Cells were incubated with $3 \mu \mathrm{M}$ DAPI in staining buffer (100 mM Tris, $\mathrm{pH} 7.4,150 \mathrm{mM} \mathrm{NaCl}, 1 \mathrm{mM} \mathrm{CaCl}, 0.5 \mathrm{mM} \mathrm{MgCl}_{2}, 0.1 \%$ Nonidet P-40) to identify nuclei and then plated onto chamber slides coated with CellTak (Corning) and allowed to settle for 2 hours at room temperature. After PBS washes, sections were mounted with ProLong Gold Antifade mountant and allowed to cure overnight in the dark at room temperature before analysis. For fluorescent staining, cardiomyocytes were permeabilized with $0.5 \%$ Triton X-100 for 15 minutes and washed with PBS. Wells were then blocked with $1 \%$ BSA in PBS for 1 hour at room temperature with subsequent incubation with anti-sarcomeric $\alpha$-actinin antibody (Abcam, catalog ab9465) overnight at $4^{\circ} \mathrm{C}$. Wells were washed with PBS and incubated with goat anti-mouse Alexa Fluor 488 (Thermo Fisher Scientific, A-10667) for 1 hour in the dark at room temperature. After PBS washes, sections were counterstained and mounted with ProLong Gold Antifade mountant with DAPI and allowed to cure overnight in the dark at room temperature before analysis. Cells were imaged at $\times 10$ or $\times 20$ magnification on an inverted wide-field fluorescence microscope (Olympus) and cardiomyocyte size was determined and number of nuclei per cardiomyocyte was counted.

Protein electrophoresis and Western blot. Tissue was homogenized in RIPA buffer with protease/phosphatase inhibitors (Thermo Fisher Scientific, 78430) diluted 1:100, and solubilized in $2 \times$ Laemmli sample buffer. Samples were heated at $80^{\circ} \mathrm{C}$ for 5 minutes with subsequent centrifugation for 5 minutes for clarity. Proteins were separated by SDS-PAGE using a $10 \%$ or $4 \%-20 \%$ Tris- $\mathrm{HCl}$ gel and transferred to a $0.45-\mu \mathrm{m}$ low-fluorescence PVDF membrane (EMD Millipore) or 0.2- $\mu$ m nitrocellulose membrane (Bio-Rad) at $100 \mathrm{~V}$ for 90 minutes at $4^{\circ} \mathrm{C}$. Membranes were subsequently blocked with $5 \%$ BSA or $5 \%$ nonfat dry milk in TBS containing $0.1 \%$ Tween-20 (TBST) and probed with primary antibody targeting MYBPC3 (catalog sc-137180), p21 (catalog sc-6246) (both Santa Cruz Biotechnology), phospho-Rb (Ser807/811, catalog 8516), Rb (catalog 9313), $\beta$-actin (catalog 8457), GAPDH (catalog 5174), or $\alpha / \beta$-tubulin (catalog 2148) (all Cell Signaling Technology) overnight at $4^{\circ} \mathrm{C}$. Membranes were washed with TBST and subsequently incubated with goat anti-mouse HRP or goat anti-rabbit HRP for 1 hour at room temperature for chemiluminescent detection. Membranes were subsequently stripped (Pierce Restore) and reprobed for detection of loading proteins. For fluorescence detection, goat anti-mouse 680LT or goat anti-rabbit $800 \mathrm{CW}$ IRDye was used with imaging on an Odyssey imaging system (Licor Biotech). Digital images of blots were then analyzed with Image Studio Lite (Licor Biotech).

$q R T-P C R$. RNA was extracted from mouse cardiac tissue using TRIzol reagent (Invitrogen) with subsequent column purification and reverse transcription (QuantiTect Reverse Transcription Kit, Qiagen) to generate cDNA. Gene-specific primers and SYBR Green reagent (Applied Biosystems) were utilized to perform the 
PCR portion of the experiment. Triplicate reactions of each sample were prepared. The $2-\Delta \Delta \mathrm{Ct}$ method was used to normalize the gene of interest to the endogenous housekeeping gene Rp132 (53). Genes with Ct values greater than 35 were considered undetectable. See Supplemental Table 1 for primer list.

Statistics. All data are displayed as mean \pm SEM unless otherwise noted. Data were analyzed using 2 -tailed Student's $t$ test for comparisons between 2 groups. For comparison between multiple groups, 1-way ANOVA was used with Tukey's post-hoc test analysis. Significance was defined as $P$ less than 0.05.

Study approval. All animal protocols and procedures were performed in accordance with NIH guidelines and approved by the Institutional Laboratory Animal Care and Use Committee at Vanderbilt University.

\section{Author contributions}

$\mathrm{BRN}, \mathrm{AFW}$, and JRB developed the research study design. BRN, AFW, AED, MSG, SCS, and JRB conducted experiments and data analysis. HSB provided reagents. BRN, AFW, HSB, and JRB prepared the manuscript.

\section{Acknowledgments}

This work was supported by grants from the NIH (K08HL116803 to J.R.B., T32HL105334 to B.R.N); Saving tiny Hearts Society (to J.R.B), the Howard Hughes Medical Institute (Medical Research Fellow awards to A.E.D.); the PhRMA Foundation (postdoctoral fellowship to S.C.S); the American Heart Association (PTF29620009 to B.R.N).

Address correspondence to: Jason R. Becker, 2220 Pierce Avenue, 342 Preston Research Building, Nashville, Tennessee 37232-6300, USA. Phone: 615.936.8297; E-mail: jason.becker@vanderbilt.edu.

1. Seidman CE, Seidman JG. Identifying sarcomere gene mutations in hypertrophic cardiomyopathy: a personal history. Circ Res. 2011;108(6):743-750.

2. Ho CY, Charron P, Richard P, Girolami F, Van Spaendonck-Zwarts KY, Pinto Y. Genetic advances in sarcomeric cardiomyopathies: state of the art. Cardiovasc Res. 2015;105(4):397-408.

3. Xin B, Puffenberger E, Tumbush J, Bockoven JR, Wang H. Homozygosity for a novel splice site mutation in the cardiac myosinbinding protein $C$ gene causes severe neonatal hypertrophic cardiomyopathy. Am J Med Genet A. 2007;143A(22):2662-2667.

4. Girolami F, et al. Clinical features and outcome of hypertrophic cardiomyopathy associated with triple sarcomere protein gene mutations. J Am Coll Cardiol. 2010;55(14):1444-1453.

5. Richard P, et al. Double heterozygosity for mutations in the beta-myosin heavy chain and in the cardiac myosin binding protein C genes in a family with hypertrophic cardiomyopathy. J Med Genet. 1999;36(7):542-545.

6. Geisterfer-Lowrance AA, et al. A mouse model of familial hypertrophic cardiomyopathy. Science. 1996;272(5262):731-734.

7. Blankenburg R, et al. $\beta$-Myosin heavy chain variant Val606Met causes very mild hypertrophic cardiomyopathy in mice, but exacerbates HCM phenotypes in mice carrying other HCM mutations. Circ Res. 2014;115(2):227-237.

8. Jiang J, Wakimoto H, Seidman JG, Seidman CE. Allele-specific silencing of mutant Myh6 transcripts in mice suppresses hypertrophic cardiomyopathy. Science. 2013;342(6154):111-114.

9. Cannon L, et al. Irreversible triggers for hypertrophic cardiomyopathy are established in the early postnatal period. J Am Coll Cardiol. 2015;65(6):560-569.

10. Mearini G, et al. Mybpc3 gene therapy for neonatal cardiomyopathy enables long-term disease prevention in mice. Nat Commun. 2014;5:5515.

11. Gedicke-Hornung C, et al. Rescue of cardiomyopathy through U7snRNA-mediated exon skipping in Mybpc3-targeted knock-in mice. EMBO Mol Med. 2013;5(7):1128-1145.

12. Li F, Wang X, Capasso JM, Gerdes AM. Rapid transition of cardiac myocytes from hyperplasia to hypertrophy during postnatal development. J Mol Cell Cardiol. 1996;28(8):1737-1746.

13. Bergmann O, Zdunek S, Alkass K, Druid H, Bernard S, Frisén J. Identification of cardiomyocyte nuclei and assessment of ploidy for the analysis of cell turnover. Exp Cell Res. 2011;317(2):188-194.

14. Richardson GD. Simultaneous assessment of cardiomyocyte DNA synthesis and ploidy: a method to assist quantification of cardiomyocyte regeneration and turnover. J Vis Exp. 2016;(111):e53979.

15. Gilsbach R, et al. Dynamic DNA methylation orchestrates cardiomyocyte development, maturation and disease. Nat Commun. 2014;5:5288.

16. Preissl S, et al. Deciphering the epigenetic code of cardiac myocyte transcription. Circ Res. 2015;117(5):413-423.

17. Jiang J, et al. Cardiac myosin binding protein C regulates postnatal myocyte cytokinesis. Proc Natl Acad Sci USA. 2015;112(29):9046-9051.

18. Ahuja P, Sdek P, MacLellan WR. Cardiac myocyte cell cycle control in development, disease, and regeneration. Physiol Rev. 2007;87(2):521-544.

19. Puente BN, et al. The oxygen-rich postnatal environment induces cardiomyocyte cell-cycle arrest through DNA damage response. Cell. 2014;157(3):565-579.

20. Mahmoud AI, et al. Meis1 regulates postnatal cardiomyocyte cell cycle arrest. Nature. 2013;497(7448):249-253.

21. Porrello ER, et al. Regulation of neonatal and adult mammalian heart regeneration by the miR-15 family. Proc Natl Acad Sci USA. 2013;110(1):187-192. 
22. Naqvi N, et al. A proliferative burst during preadolescence establishes the final cardiomyocyte number. Cell. 2014;157(4):795-807.

23. LaBaer J, et al. New functional activities for the p21 family of CDK inhibitors. Genes Dev. 1997;11(7):847-862.

24. Zhang H, Hannon GJ, Beach D. p21-containing cyclin kinases exist in both active and inactive states. Genes Dev. 1994;8(15):1750-1758.

25. Fox DT, Duronio RJ. Endoreplication and polyploidy: insights into development and disease. Development. 2013;140(1):3-12.

26. Lee HO, Davidson JM, Duronio RJ. Endoreplication: polyploidy with purpose. Genes Dev. 2009;23(21):2461-2477.

27. Duncan AW, et al. Ploidy reductions in murine fusion-derived hepatocytes. PLoS Genet. 2009;5(2):e1000385.

28. Orr-Weaver TL. When bigger is better: the role of polyploidy in organogenesis. Trends Genet. 2015;31(6):307-315.

29. Zimmet J, Ravid K. Polyploidy: occurrence in nature, mechanisms, and significance for the megakaryocyte-platelet system. Exp Hematol. 2000;28(1):3-16.

30. Cross JC. Genetic insights into trophoblast differentiation and placental morphogenesis. Semin Cell Dev Biol. 2000;11(2):105-113.

31. Losick VP, Fox DT, Spradling AC. Polyploidization and cell fusion contribute to wound healing in the adult Drosophila epithelium. Curr Biol. 2013;23(22):2224-2232.

32. Diril MK, et al. Cyclin-dependent kinase $1(\mathrm{Cdk} 1)$ is essential for cell division and suppression of DNA re-replication but not for liver regeneration. Proc Natl Acad Sci USA. 2012;109(10):3826-3831.

33. Adachi S, et al. Programmed induction of endoreduplication by DNA double-strand breaks in Arabidopsis. Proc Natl Acad Sci USA. 2011;108(24):10004-10009.

34. Xiao G, et al. Inducible activation of c-Myc in adult myocardium in vivo provokes cardiac myocyte hypertrophy and reactivation of DNA synthesis. Circ Res. 2001;89(12):1122-1129.

35. Pasumarthi KB, Nakajima H, Nakajima HO, Soonpaa MH, Field LJ. Targeted expression of cyclin D2 results in cardiomyocyte DNA synthesis and infarct regression in transgenic mice. Circ Res. 2005;96(1):110-118.

36. Chaudhry HW, et al. Cyclin A2 mediates cardiomyocyte mitosis in the postmitotic myocardium. J Biol Chem. 2004;279(34):35858-35866.

37. Soonpaa $\mathrm{MH}$, et al. Cyclin D1 overexpression promotes cardiomyocyte DNA synthesis and multinucleation in transgenic mice. J Clin Invest. 1997;99(11):2644-2654.

38. Tane S, et al. Repression of cyclin D1 expression is necessary for the maintenance of cell cycle exit in adult mammalian cardiomyocytes. J Biol Chem. 2014;289(26):18033-18044.

39. Diehl JA, Cheng M, Roussel MF, Sherr CJ. Glycogen synthase kinase-3beta regulates cyclin D1 proteolysis and subcellular localization. Genes Dev. 1998;12(22):3499-3511.

40. Alt JR, Cleveland JL, Hannink M, Dieh1 JA. Phosphorylation-dependent regulation of cyclin D1 nuclear export and cyclin D1-dependent cellular transformation. Genes Dev. 2000;14(24):3102-3114

41. McConnell BK, et al. Dilated cardiomyopathy in homozygous myosin-binding protein-C mutant mice. J Clin Invest. 1999;104(9):1235-1244.

42. Wang JJ, et al. Genetic dissection of cardiac remodeling in an isoproterenol-induced heart failure mouse model. PLoS Genet. 2016;12(7):e1006038.

43. Barnabei MS, Palpant NJ, Metzger JM. Influence of genetic background on ex vivo and in vivo cardiac function in several commonly used inbred mouse strains. Physiol Genomics. 2010;42A(2):103-113.

44. Leferovich JM, et al. Heart regeneration in adult MRL mice. Proc Natl Acad Sci USA. 2001;98(17):9830-9835.

45. Kempermann G, Brandon EP, Gage FH. Environmental stimulation of 129/SvJ mice causes increased cell proliferation and neurogenesis in the adult dentate gyrus. Curr Biol. 1998;8(16):939-942.

46. Chen PP, Patel JR, Powers PA, Fitzsimons DP, Moss RL. Dissociation of structural and functional phenotypes in cardiac myosin-binding protein C conditional knockout mice. Circulation. 2012;126(10):1194-1205

47. Merkulov S, Chen X, Chandler MP, Stelzer JE. In vivo cardiac myosin binding protein C gene transfer rescues myofilament contractile dysfunction in cardiac myosin binding protein C null mice. Circ Heart Fail. 2012;5(5):635-644.

48. Faul F, Erdfelder E, Lang AG, Buchner A. G*Power 3: a flexible statistical power analysis program for the social, behavioral, and biomedical sciences. Behav Res Methods. 2007;39(2):175-191.

49. Skarnes WC, et al. A conditional knockout resource for the genome-wide study of mouse gene function. Nature. 2011;474(7351):337-342.

50. Hougen $\mathrm{K}$, et al. Cre-loxP DNA recombination is possible with only minimal unspecific transcriptional changes and without cardiomyopathy in Tg(alphaMHC-MerCreMer) mice. Am J Physiol Heart Circ Physiol. 2010;299(5):H1671-H1678.

51. Lao Z, Raju GP, Bai CB, Joyner AL. MASTR: a technique for mosaic mutant analysis with spatial and temporal control of recombination using conditional floxed alleles in mice. Cell Rep. 2012;2(2):386-396.

52. Murray TV, et al. Redox regulation of cardiomyocyte cell cycling via an ERK1/2 and c-Myc-dependent activation of cyclin D2 transcription. J Mol Cell Cardiol. 2015;79:54-68.

53. Brattelid T, Winer LH, Levy FO, Liestø1 K, Sejersted OM, Andersson KB. Reference gene alternatives to Gapdh in rodent and human heart failure gene expression studies. BMC Mol Biol. 2010;11:22. 